\title{
Muertes por incidentes viales en Bello (Antioquia) (2012-2016)*
}

\author{
Deaths due to road incidents in Bello (Antioquia) (2012-2016)
}

\author{
Mortes por acidentes rodoviários em Bello (Antioquia) (2012-2016)
}

\author{
Luisa Fernanda Marroquín-Muñoz'; Hugo Grisales-Romero². \\ 1 Profesional en Gerencia de Sistemas de Información en Salud, Universidad de Antioquia. Colombia. Correo electrónico: luisafdamarro@ \\ gmail.com; ORCID: https://orcid.org/0000-0001-6733-1902. \\ 2 Doctor en Epidemiología, Grupo Demografía y Salud, Universidad de Antioquia. Colombia. Correo: hugo.grisales@udea.edu.co. \\ ORCID: https://orcid.org/0000-0001-5493-0009.
}

Recibido: 20/11/2018. Aprobado: 06/08/2019. Publicado: 01/10/2019

Marroquín-Muñoz LF, Grisales-Romero H. Muertes por incidentes viales en (Antioquia) (2012-2016). Rev. Fac. Nal. Salud Pública. 2019;37(3):86-97. DoI:10.17533/udea.rfnsp.v37n3a10

\section{Resumen}

Objetivo: Determinar la estructura de la mortalidad por incidentes viales en el municipio de Bello (Antioquia), entre los años 2012 y 2016. Metodología: Estudio descriptivo, con información obtenida de las bases de datos aerca de defunciones, parque automotor y malla vial del municipio. Se calcularon frecuencias y tasas de mortalidad para las variables pertinentes; con el análisis de correspondencias múltiples se determinó el perfil del fallecido, y para conocer la distribución espacial de las muertes se utilizó un mapa de densidad de Kernel, según el método de clasificación estándar cuantil. Resultados: Se registraron 313 defunciones por incidentes viales, 63 en promedio por año (13,4 por cada 100000 habitantes), específicamente en los hombres (62 vs. 12 mujeres por 100000$)$, que tenían entre 19 y 28 años (47,9\%), con básica primaria $(32,5 \%)$ y que no estaban asegurados (44\%).
Predominaron los incidentes viales por choques $(53,0 \%)$ y atropellamientos (41\%), los fines de semana y entre las 0:00 y las 6:00 a. m. (29,2\%); fueron las motocicletas las que más incidentes viales generaron con muertes $(74,4 \%)$. Las vías de mayor concentración de víctimas fatales fueron: en una parte de la Autopista Norte —entre las diagonales 50 y 52 $(14,0 \%)$, la calle $46(2,2 \%)$ y la carrera $50(5,1 \%)$ - y la Autopista Medellín-Bogotá (5,1\%), y en las comunas Suárez y Niquía (2,37 y 1,78 por cada 100 000). Conclusiones: Dado el importante aumento de la incidentalidad vial y, en consecuencia, de las muertes que de ella se deriva, se interroga acerca de la efectividad de las intervenciones emprendidas y se sugiere su valoración.

--------Palabras clave: incidente vial, accidentes, muertes violentas, delitos de tránsito, Colombia (fuente: Tesauro de política criminal latinoamericana - ILANUD) 


\section{Abstract}

Objective: To determine the structure of mortality due to road incidents in the municipality of Bello (Antioquia), from 2012 to 2016. Methodology: Descriptive study with information obtained from the databases about deaths, the automotive fleet, as well as the road network in the municipality. Mortality rates for relevant variables were calculated. Also, the profile of a deceased person was determined via multiple correspondence analysis. Furthermore, the spatial distribution of deaths was found through a Kernel density estimation map according to the quantile standard classification method. Results: 313 deaths caused by road incidents were registered. 63 per year on average (being 13.4 per 100,000 inhabitants). This reality was specifically observed in men (62 vs. 12 women per 100,000), who were 19 to 28 years old $(47.9 \%)$, had primary school education $(32.5 \%)$, and did not have insurance (44\%). Most road incidents were crashes (53\%) and run-overs (41\%), on weekends and from 0:00 to 6:00 a.m. (29.2\%). Motorcycles caused the most fatal road incidents (74.4 $\%)$. The routes with the highest concentration of fatalities were: a segment of the Autopista Norte - between diagonales 50 and 52 (14\%), Calle 46 (2.2\%) and Carrera 50 (5.1\%)_- Avenida Medellín-Bogotá (5.1\%), and in the Suárez and Niquía areas (2.37 and 1.78 per 100,000 inhabitants). Conclusions: Given the significant increase of road incidents - and therefore deaths linked to it - the effectiveness of interventions performed so far is brought into question and a reassessment of those interventions is suggested.

--Keywords: road incident, accidents, violent deaths, traffic offenses, Colombia (source: Thesaurus of Latin American Criminal Policy - ILANUD).

\section{Resumo}

Objetivo: Determinar a estrutura de mortalidade por incidentes rodoviários no município de Bello (Antioquia), entre 2012 e 2016. Metodologia: Estudo descritivo, com informações obtidas nas bases de dados atinentes aos óbitos, parque automotivo e rede viária do município. As frequências e taxas de mortalidade foram mensuradas para as variáveis salientadas; com a análise de correspondência múltipla, foi determinado o perfil do falecido e, para conhecer a distribuição espacial das mortes, foi utilizado um mapa de densidade de Kernel, de acordo com o método padrão de classificação quantílica. Resultados: foram registradas 313 mortes por acidentes de trânsito, 63 em média por ano (13,4 por 100.000 habitantes), especificamente em homens (62 vs. 12 mulheres por 100.000), com idades entre 19 e 28 anos (47,9\%), com ensino fundamental básico $(32,5 \%)$ e não segurados (44\%).
Os incidentes nas estradas predominaram por acidentes inter vehiculares $(53,0 \%)$ e atropelamentos $(41 \%)$, nos finais de semana e entre as 0:00 e as 6:00 da manhã (29,2\%); foram as motos que geraram mais incidentes nas estradas com mortes $(74,4$ \%). As rotas com maior concentração de mortes foram: em uma parte da Rodovia Norte - entre as 50 e 52 diagonais $(14,0 \%)$, a rua $46(2,2 \%)$ e a rua $50(5,1 \%)$ - Rodovia MedellínBogotá (5,1\%), e nas comunas de Suárez e Niquía (2,37 e 1,78 por cada 100 000). Conclusões: Dado o aumento significativo da incidentalidade nas rodovias e, consequentemente, das mortes resultantes, questiona-se a eficácia das intervenções realizadas e sugere-se sua avaliação.

-Palavras-chave: incidentes nas estradas, acidentes, mortes violentas, infrações de trânsito, Colômbia (fonte: Thesaurus da política criminal na América Latina) - ILANUD).

\section{Introducción}

En Colombia, el Código Nacional de Tránsito Terrestre define "accidente" como un "evento generalmente involuntario, generador al menos por un vehículo en movimiento, que causa daños a las personas y bienes involucrados en él" [1]. Sin embargo, Bonilla y Gutiérrez, en 2014, argumentan que, en las últimas décadas, "la comprensión de los factores que determinan la naturaleza de las lesiones ha cambiado este concepto $\mathrm{y}$ ha hecho que el término 'accidente' sea inexacto y, en consecuencia, las lesiones que de él se deriven son eventos prevenibles" [2; traducción nuestra]. Espinosa, Cabrera y Velásquez, en 2017, comparten la apreciación anterior y definen el "incidente vial" como

[...] un evento que trasfiere en una fracción de tiempo energía cinética entre superficies inertes y vivas durante la movilidad. Dicha transferencia tiene potencial de lesionar fatalmente o no a personas de toda edad que se desplazan a pie, patines, bicis u otros medios sin motor o motorizados, desde o al hogar, trabajo, estudio o sitios de recreación, en fin, dónde ocurre la vida [...]. [Los incidentes viales] no dependen del azar o suerte y, por tanto, no son accidentes tal como Naciones Unidas lo señaló hace décadas. Son controlables como riesgo, prevenibles como exposición, evitables en ocurrencia $\mathrm{y}$ atenuables en sus efectos individual, familiar y social cuando es imposible prevenirlos [3, p. 8].

Así, se concluye que un accidente vial refiere una situación que no es prevenible, mientras el incidente vial sí lo es, con el agravante que este refleja la actuación de las personas cuando están en frente del volante.

En ese sentido, en este artículo se acogen los planteamientos que rebaten las definiciones clásicas de accidente y se asume el incidente vial como un suceso inesperado sobre la vía pública, ocasionado al menos por un vehículo en movimiento con o sin motor, que 
generalmente produce daños, en muertes o en lesionados, y que genera pérdidas socioeconómicas que afectan a la seguridad vial de la población, cuya característica peculiar es que es previsible, prevenible y controlable.

Los traumatismos ocasionados por los incidentes viales son un problema de salud pública: cada día mueren en el mundo más de 3800 personas; en promedio, por año, hay 1,25 millones de víctimas, y entre 20 y 50 millones presentan traumatismos no letales. Más del $90 \%$ de las defunciones se producen en los países de ingresos bajos y medianos, donde se ubican aproximadamente el $54 \%$ de los vehículos del mundo. El $50 \%$ de las víctimas mortales por lesiones ocasionadas en un incidente vial son peatones, ciclistas y motociclistas, usualmente con edades entre 15 y 29 años [4-6].

La Organización Mundial de la Salud (OMS) prevé que, para el año 2030, los incidentes viales serán la quinta causa de mortalidad, dejando como resultado unos 2,4 millones de víctimas mortales al año. Las pérdidas a nivel mundial por incidentes viales se han estimado en US\$ 518000 millones anuales, de los cuales US\$ 65000 millones corresponden a los países de ingresos bajos y medianos, más que la cantidad total que reciben estos países por concepto de asistencia para el desarrollo. En particular, los costos derivados de los traumatismos letales y no letales ascienden al $1 \%$ del producto interno bruto en los países de ingresos bajos, al $1,5 \%$ en los de ingresos medianos y al $2 \%$ en los de ingresos altos [4-6].

En Colombia, los incidentes viales dejan más de 7000 muertos al año y son la segunda causa de muerte violenta en el país. En 2016, el Instituto Nacional de Medicina Legal y Ciencias Forenses registró 7280 víctimas fatales por incidentes viales y 45256 personas lesionadas o discapacitadas; la mortalidad por incidentes viales fue de 14,93 por cada 100000 habitantes, muy cercana a la tasa de la región de las Américas $(15,9)$ [7]. Las tasas más altas se presentaron en los departamentos del Casanare, Arauca y Meta, y en los municipios de Bogotá, Cali y Medellín. El número de muertes ocurridas por incidentes viales ese año fue la cifra más alta en el siglo XXI y en particular la mayor de la última década [7], lo que retrasa al país en el cumplimiento del objetivo del decenio de acción para la seguridad vial 2011-2020 (-50\%, 2852 muertes en el 2020) [8], del Plan Nacional de Seguridad Vial (-26 \%, 4224 muertes en el año 2021) [9] y de las metas sobre seguridad vial propuestas por los "Objetivos de Desarrollo Sostenible" de la Organización de las Naciones Unidas [10].

En el municipio de Bello (Antioquia), en el año 2016, las defunciones por incidentes viales ocuparon el séptimo lugar entre las diez primeras causas de muerte, según la Secretaría de Salud de dicho municipio, posicionándose como el segundo municipio del departamento con el índice más alto de mortalidad por la causa enunciada. Las víctimas fatales por incidentes viales han aumentado en los últimos diez años, afectando a la población más joven y vulnerable de la vía pública, problemática que se explica por factores relacionados con la cultura ciudadana, por la existencia de medidas flexibles que propician conductas indebidas en la vía pública y por ser un municipio con vías arterias con importante flujo vehicular, entre otros [11]. Las situaciones mencionadas perturban la seguridad, el orden y la movilidad vial, pero la magnitud del problema se desconoce para el municipio, lo que sustenta la prioridad de caracterizar la mortalidad por incidentes viales en general y su georreferenciación entre los años 2012 y 2016, con el fin de diseñar estrategias de intervención, con sustento científico, que se encaminen a mejorar la seguridad vial.

\section{Metodología}

Este artículo refiere la experiencia de un estudio descriptivo, basado en fuentes secundarias, donde se aborda una problemática que ocasiona pérdidas humanas y económicas, a pesar de ser un evento prevenible y evitable: la incidentalidad vial en el municipio de Bello, entre los años 2012 y 2016.

La población de estudio fueron todas las personas que fallecieron en el periodo de tiempo indicado, registradas en los certificados individuales de defunción del Departamento Administrativo Nacional de Estadística (DANE) y en la base de datos del Sistema Integral de Información de la Protección Social. Además, la Secretaría de Movilidad del municipio de Bello facilitó la información relacionada con el parque automotor y las muertes ocurridas en ese periodo de tiempo.

La unidad de análisis fue cada defunción, la cual, según la "Clasificación estadística internacional de enfermedades y problemas relacionados con la salud. Décima Revisión. CIE-10), correspondió a accidentes de transporte, y de acuerdo con la Lista 6/67-CIE-10 oms/ops [Organización Panamericana de la Salud], que refería las defunciones por causas externas [12,13].

Además, se requirió de las proyecciones censales del DANE, de los mapas de comuna-barrio y de la malla vial del municipio, información que fue descargada de la página web del Instituto Geográfico Agustín Codazzi. Esto permitió ubicar el lugar de ocurrencia de los incidentes con muertes.

La obtención del barrio y la comuna donde se presentó el desenlace se hizo confrontando la dirección de ocurrencia de la defunción con la información de la malla vial del municipio de Bello. Las direcciones que no coincidían con la división político-administrativa del municipio fueron consultadas en el servidor de 
aplicaciones de mapas en la web, Google Maps, y cuando se presentaron inconsistencias, se descartaron del análisis.

Se realizó la estimación del subregistro de las defunciones con el método de Preston y Coale (1978), lo que facilitó la obtención de una base de datos ajustada por sexo y edad, la cual cumplió con las características de calidad y coherencia, permitiendo, además, el control de los sesgos de selección e información.

En el análisis estadístico se construyeron tablas de frecuencia para las variables de persona y tiempo, tasas de mortalidad por cada 100000 habitantes y 10000 vehículos, y los indicadores de resumen para las variables cuantitativas. Se utilizó el análisis de correspondencias múltiple con el fin de caracterizar el perfil del fallecido por incidentes viales, por medio de mapas perceptuales, a través de relaciones de cercanía y lejanía entre las categorías de los factores. Para el análisis geográfico se procedió a construir la distribución espacial de las muertes por incidentes viales según la dirección de ocurrencia del desenlace fatal, que se estandarizó según criterios de geocodificación con uso del software ArcGIS ${ }^{\circledR}$.

El porcentaje de direcciones geocodificadas y ubicadas en la malla vial fue del $80 \%$; las no encontradas fueron revisadas, una por una, con el fin de detectar posibles errores, lo que posibilitó que se aumentara el porcentaje anterior en un $16 \%$.

Al obtener la ubicación espacial de la ocurrencia de cada muerte, a través de un mapa de densidad de Kernel, se visualizaron las zonas de alta y baja densidad [14]. Luego se observaron las direcciones con más víctimas fatales por incidentes viales, por medio de un mapa temático con simbología por atributos múltiples, que permitió establecer las vías con más incidentes viales con muertes. Una vez se realizó este procedimiento, se identificó el barrio y posteriormente la comuna. Mediante uso del software ArcGIS ${ }^{\circledR}$, se construyeron los mapas temáticos según la distribución de la tasa ajustada de mortalidad general por incidentes viales por comuna y de la tasa mediana de mortalidad general por incidentes viales por barrio, entre los años 2012 y 2016.

Para la representación de las tasas de mortalidad por incidentes viales en los barrios y las comunas, se tuvo en cuenta el método de clasificación estándar "cuantil", que asigna el mismo número de valores de datos a cada clase, y donde no existen clases vacías ni clases con excesos o pocos valores [15]. Este método distribuyó los intervalos de las tasas de mortalidad general por incidentes viales, de forma que cada clase tuviera un número igual entre cada leyenda de los mapas, con lo que se identificó el barrio y las comunas con mayores eventos.

Se siguieron los aspectos éticos formalizados en la Resolución 8430 de 1993 del Ministerio de Salud [16], la Ley Estatutaria 1581 de 2012 [17] y el Decreto 1377 de 2013 [18], que asegura el buen manejo de los datos para el presente estudio. Para la estructuración de las bases de datos y el análisis subsiguiente, se utilizaron los programas Excel $\AA$, IBM SPSS Statistics $21 \AA$, ArcGIS 10®, y Microsoft Word $\AA$.

\section{Resultados}

En el municipio de Bello, en el periodo comprendido entre los años 2012 y 2016 se registraron 313 defunciones por incidentes viales, 63 en promedio por año. La tasa mediana de mortalidad general en el periodo fue de $13,4^{\dagger}$ por cada 100000 habitantes, aproximadamente.

Las defunciones prevalecieron en los hombres, en el $83 \%$ de los casos, y en promedio, por año, se registraron 52 muertes en los hombres y 11 en las mujeres.

En el año central del periodo (2014), por cada 100000 hombres se presentaron 24,5 muertes, y en las mujeres, 3,0 por cada 100000 mujeres (véase Tabla 1).

Tabla 1. Distribución de frecuencias de las defunciones y de la mortalidad general por incidentes viales según sexo. Bello, 2012-2016

\begin{tabular}{cccccccccc}
\hline \multirow{2}{*}{ Año } & \multicolumn{3}{c}{ Hombres } & \multicolumn{3}{c}{ Mujeres } & \multicolumn{3}{c}{ Total } \\
\cline { 2 - 9 } & Defunciones* $^{*}$ Tasa** & $\%$ & Defunciones* $^{*}$ & Tasa** & $\%$ & Defunciones* $^{\text {Tasa** }}$ & $\%$ \\
\hline 2012 & 55 & 26,5 & 21,2 & 15 & 6,8 & 27,8 & 70 & 16,3 & 22,4 \\
2013 & 55 & 25,9 & 21,2 & 11 & 4,9 & 20,4 & 66 & 15,0 & 21,1 \\
2014 & 53 & 24,5 & 20,5 & 7 & 3,0 & 13,0 & 60 & 13,4 & 19,2 \\
2015 & 52 & 23,6 & 20,1 & 7 & 3,0 & 13,0 & 59 & 12,9 & 18,8 \\
2016 & 44 & 19,6 & 17,0 & 14 & 5,8 & 25,8 & 58 & 12,5 & 18,5 \\
\hline Total & 259 & & 100,0 & 54 & & 100,0 & 313 & & 100,0 \\
\hline
\end{tabular}

* Defunciones ajustadas por subregistro.

** Tasa por 100.000 habitantes

† Obtenida de dividir la mediana de las muertes y la mediana de la población. 
El promedio de edad de los fallecidos fue de 42 años (Desviación estándar $-\mathrm{DE}-=21,9$ años) y la frecuencia más alta de presentación fue a los 23 años. Las tasas de mortalidad más altas predominaron en los jóvenes de 19 a 28 años de edad, seguidos por los adolescentes de 12 a 18 años [19] (92,3 y 56,8 por cada 100000 habitantes, respectivamente). Nótese que por cada mujer fallecida por causa de un incidente vial, aproximadamente 5 hombres murieron por la misma causa.

Cuando se consideró la distribución de la mortalidad por grupos de edad y sexo, esta fue superior en los hombres y en las mujeres entre 19 y 28 años (17 y 2,1 por cada 10000 habitantes, respectivamente). En los hombres y en las mujeres de máximo 11 años, la magnitud de la mortalidad por incidentes viales fue menor $(1,1 \mathrm{y}$ 0,5 por cada 10000 habitantes, respectivamente).

Los occisos, en su mayoría, eran solteros (47,9\%); tenían nivel educativo máximo de básica primaria $(32,5 \%)$ y al momento del incidente vial no tenían afiliación a la seguridad social (44\%). El 48,3\% de las defunciones se concentró en los últimos cinco meses de todos los años, especialmente en septiembre $(10,2 \%)$, que contrasta con las del mes de abril (5,4\%). En los fines de semana, en los días sábado y domingo, las frecuencias de presentación de las muertes fueron mayores (15,7 y 19,5\%, en ese orden), en contraste con el día miércoles $(9,9 \%)$. En general, el 29,2\% de las muertes ocurrieron entre las 0:00 horas y las 6:00 a. m., siendo más asidua la ocurrencia del hecho fatal entre las 0:00 horas y las 2:00 a. m. (15,1\%) y de las 18:00 horas a las 20:00 horas (12,8\%) (véase Figura 1).

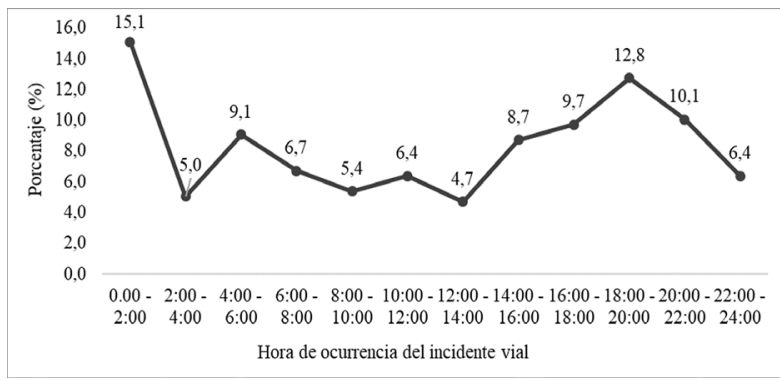

Figura 1. Distribución porcentual de las defunciones por incidentes viales según hora de ocurrencia. Bello, 20122016.
Defunciones/mortalidad según la condición de la víctima, la clase de incidente y el tipo de vehículo donde ocurrió el evento

En general, en el periodo, se presentaron 364 incidentes viales con muertes por cada 10000 vehículos y, en particular, por cada 27 vehículos se registró una persona fallecida. En el periodo, el número de incidentes viales varió entre 358 y 457 por cada 10000 vehículos, con una tendencia ascendente, de año a año, destacándose que fue mayor en 2013.

La tasa mediana de mortalidad general por incidentes viales fue mayor cuando el tipo de vehículo era de categoría 2 , con 28,8 muertes por cada 100 vehículos y la más baja fue para aquellas de categoría 3 y 5 , con 0,6 y 0,4 muertes respectivamente por cada 100 vehículos (véase Tabla 2).

En el quinquenio, las defunciones se concentraron en los conductores $(35,5 \%)$ y los peatones $(30,0 \%)$. En promedio, por año se registraron 22,2 muertes en los conductores ( $\mathrm{DE}=2,3$ muertes) y 18,8 en los peatones $(\mathrm{DE}=3,8$ muertes $)$. Predominaron las muertes de conductores y peatones hombres por incidentes viales $(52,7$ y $39,9 \%)$, a diferencia de las mujeres, que eran principalmente peatonas.

Fue mayor la frecuencia de muertes para los conductores que tuvieron choques o volcamientos, y para los peatones que fueron atropellados. Del total de muertes debidas a los choques, quienes llevaron la peor parte fueron los conductores $(78,7 \%)$, y los pasajeros (16,5\%).

Con respecto al total de atropellos, se destacó que el $96,9 \%$ fueron en peatones y el $1 \%$ en personas que estaban en el vehículo involucrado en el evento:.

En el grupo de edad de 19 a 28 años prevalecieron más las muertes para los conductores y pasajeros, y después de los 29 años, para los peatones.

Predominaron las muertes en los conductores que manejaban bicicletas o motocicletas, en los pasajeros que iban en un automóvil/taxi/campero/camioneta, y en los peatones atropellados por vehículos de cuatro o más llantas (véase tabla 2).

Entre 2012 y 2016, el $53 \%$ de las defunciones fueron por choques, el $41 \%$ debido a atropellos y el $6 \%$ restante a volcamientos; en promedio, por año, fallecieron 26 personas debido a choques, 20 a atropellos y 3 a volcamientos.

La tasa mediana de mortalidad general por incidentes viales en el periodo fue mayor cuando el evento principal fue un choque (6 por cada 100000 ), la que predominó en los hombres, (52,3 por cada 100000$)$, un poco más de

\footnotetext{
De acuerdo con la Real Academia Española, el verbo “atropellar”, en su tercera acepción, refiere la acción de un medio de transporte que alcanza "violentamente a personas o animales, chocando con ellos y ocasionándoles, por lo general, daños"; "chocar", por su parte, es un encuentro violento entre dos cosas — no necesariamente entre una persona y una cosa - [20]. Así, pues, cuando un peatón es alcanzado violentamente por un vehículo, no se puede hablar de choque o colisión, sino simplemente de "atropello"
} 
Tabla 2. Distribución de las defunciones, de la calidad del fallecido, del parque automotor y de la mortalidad general por incidentes viales según tipo de vehículo. Bello, 2012-2016.

\begin{tabular}{|c|c|c|c|c|c|c|c|c|c|c|c|c|}
\hline \multirow{2}{*}{ Tipo de vehículo } & \multicolumn{2}{|c|}{ Defunciones } & \multicolumn{2}{|c|}{ Conductor } & \multicolumn{2}{|c|}{ Pasajero } & \multicolumn{2}{|c|}{ Peatón } & \multicolumn{2}{|c|}{$\begin{array}{l}\text { Sin infor- } \\
\text { mación }\end{array}$} & \multirow[t]{2}{*}{$\begin{array}{c}\text { Parque au- } \\
\text { tomotor* }^{*}\end{array}$} & \multirow[t]{2}{*}{ Tasa** } \\
\hline & $\mathrm{n}$ & (\%) & $\mathrm{n}$ & $(\%)$ & $\mathrm{n}$ & (\%) & $\mathrm{n}$ & $(\%)$ & $\mathrm{n}$ & $(\%)$ & & \\
\hline $\begin{array}{l}\text { Categoría } 1 \\
\text { (Bicicletas) }\end{array}$ & 9 & 2,9 & 9 & 100,0 & 0 & 0,0 & 0 & 0,0 & 0 & 0,0 & SD & $\mathrm{SD}$ \\
\hline $\begin{array}{l}\text { Categoría } 2 \\
\text { (Motocicletas) }\end{array}$ & 180 & 57,5 & 101 & 56,1 & 25 & 13,9 & 48 & 26,7 & 6 & 3,3 & 125 & 28,8 \\
\hline $\begin{array}{l}\text { Categoría } 3 \\
\text { (Automóviles, } \\
\text { taxis, camperos y } \\
\text { camionetas) }\end{array}$ & 23 & 7,3 & 1 & 4,3 & 4 & 17,4 & 18 & 78,3 & 0 & 0,0 & 802 & 0,6 \\
\hline $\begin{array}{l}\text { Categoría } 4 \\
\text { (Buses, busetas y } \\
\text { microbuses) }\end{array}$ & 20 & 6,4 & 0 & 0,0 & 2 & 10,0 & 18 & 90,0 & 0 & 0,0 & 120 & 3,3 \\
\hline $\begin{array}{l}\text { Categoría } 5 \\
\text { (Camiones, } \\
\text { volquetas y } \\
\text { tractocamiones) }\end{array}$ & 10 & 3,2 & 0 & 0,0 & 0 & 0,0 & 10 & 100,0 & 0 & 0,0 & 533 & 0,4 \\
\hline Sin información & 71 & 22,7 & 0 & 0,0 & 0 & 0,0 & 0 & 0,0 & 71 & 100,0 & SD & $\mathrm{SD}$ \\
\hline Total & 313 & 100,0 & 111 & 35,5 & 31 & 9,9 & 94 & 30,0 & 77 & 24,6 & & \\
\hline
\end{tabular}

seis veces que la de las mujeres $(8,2$ por cada 100000$)$, en quienes los atropellos tuvieron un papel protagónico (8,7 por cada 100000$)$.

La mortalidad más alta, por choques y volcamientos, ocurrió en personas entre 19 y 28 años (61,2 por cada 100000 y 6 por cada 100000 , respectivamente), seguida por la ocasionada por atropellos, en el grupo de edad de 29 a 59 años (22,3 por cada 100000$)$.

Perfil del fallecido y distribución espacial de las muertes

Especialmente en el mes de enero, los solteros y los que vivían en unión libre, que tenían un nivel educativo alto y estaban adscritos al régimen contributivo, fallecieron a causa de un incidente vial en la vía pública. Con respecto a los conductores y pasajeros fallecidos por choques cuando conducían bicicletas o motocicletas, tenían entre 19 y 28 años, cuyos decesos ocurrieron especialmente el día domingo, en los meses de marzo y octubre.

Las zonas de mayor concentración de las víctimas fatales en el municipio de Bello, entre 2012 y 2016, fueron en las autopistas Norte y Medellín-Bogotá, vías de mayor circulación de vehículos y de gran importancia, porque conectan al municipio con otros territorios que son altamente productivos, en la industria, comercio, turismo y agricultura (véase Figura 2).
La vía de mayor concentración de víctimas fatales por incidentes viales fue la Autopista Norte (21,3\%), que se divide entre la diagonal 52, con el 7,3\%, la diagonal 50 y 51 (por la rotonda de la Estación Niquía del Metro), con el $6,7 \%$, la calle 46 , por el sector de Cotrafa, y la carrera 50 (por la rotonda de la Estación Madera), con el 5,1 \% de víctimas fatales. La Autopista Medellín-Bogotá fue la otra zona de importante incidentalidad, con víctimas fatales, con el 5,1\% de las muertes (véase figura 3).

\section{Georreferenciación de la mortalidad según} comuna de ocurrencia del incidente vial

En el municipio de Bello, en el periodo comprendido entre los años 2012 y 2016, la mayor tasa ajustada de mortalidad general por incidentes viales se presentó en la comuna 4 (Suárez) (2,37 por cada 100 000), seguido por la comuna 8 (Niquía) (1,78 por cada 100 000) y la comuna 11 (Zamora) (1,68 por cada 100 000), en las cuales se concentró el 57,1 \% de los desenlaces. También en la comuna 2 (La Madera), comuna 9 (Guasimalito) y la comuna 5 (La Cumbre) hubo alto número de muertes por cada 100000 habitantes, de 1,43 por cada 1000001,09 por cada 100000 y 0,51 por cada 100000 en ese orden, que representaron el 30,4\% de las mismas. En la comuna 6 (Bellavista), comuna 7 (Altos de Niquía), comuna 10 (Fontidueño) y comuna 1 
(París), la mortalidad por incidentes viales fue la más baja, pues varió entre 0,12 por cada 100000 y 0,33 por cada $100000 \mathrm{La}$ mayor tasa mediana de mortalidad general por incidentes viales se presentó en el barrio La Navarra $(0,67$ por cada 100000$)$, ubicado en la comuna Guasimalito (véase Figura 4)

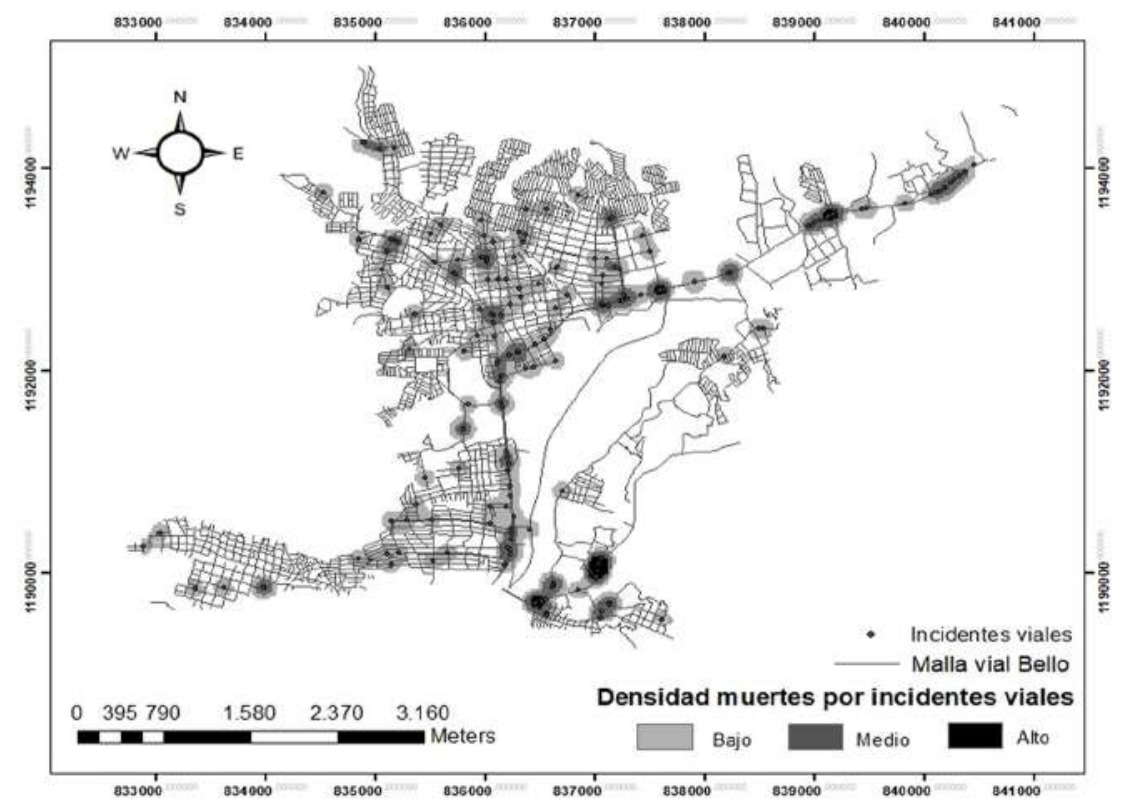

Figura 2. Densidad de las muertes por incidentes viales en el municipio de Bello, 2012-2016.

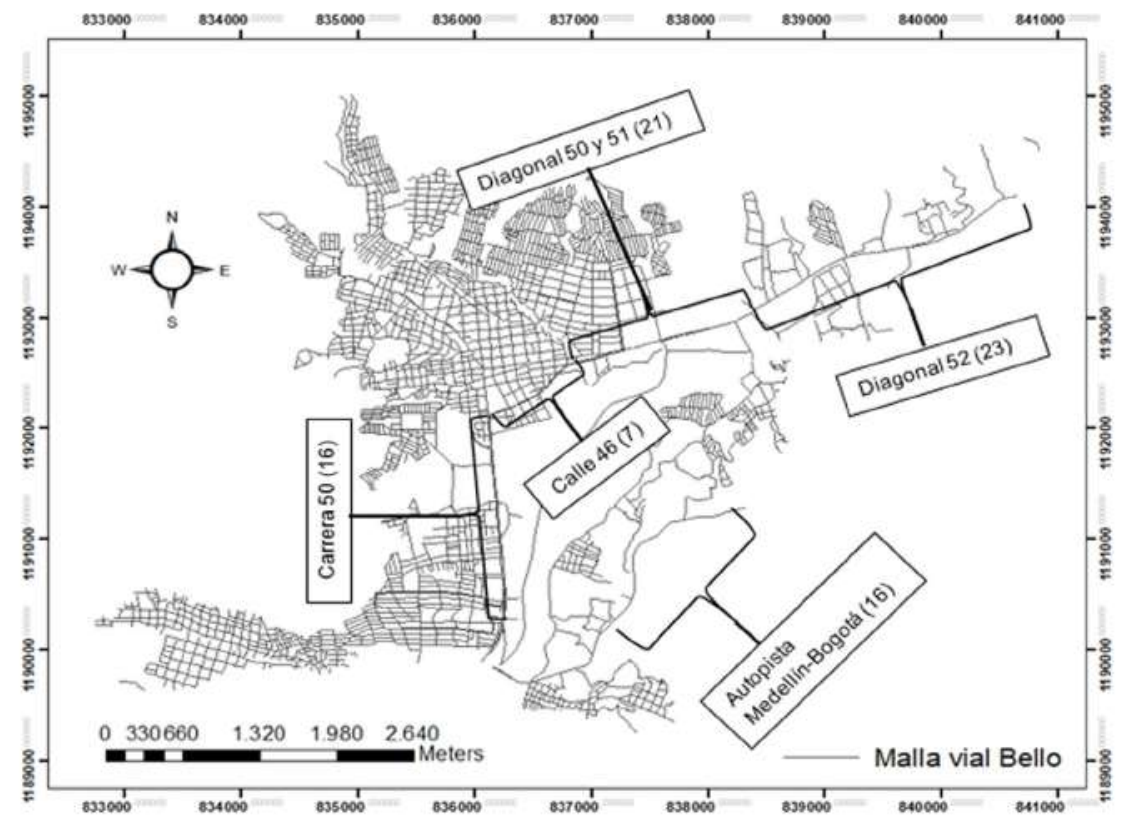

Figura 3. Vías de mayor frecuencia de muertes por incidentes viales y cantidad de muertes. Bello, 2012-2016. 


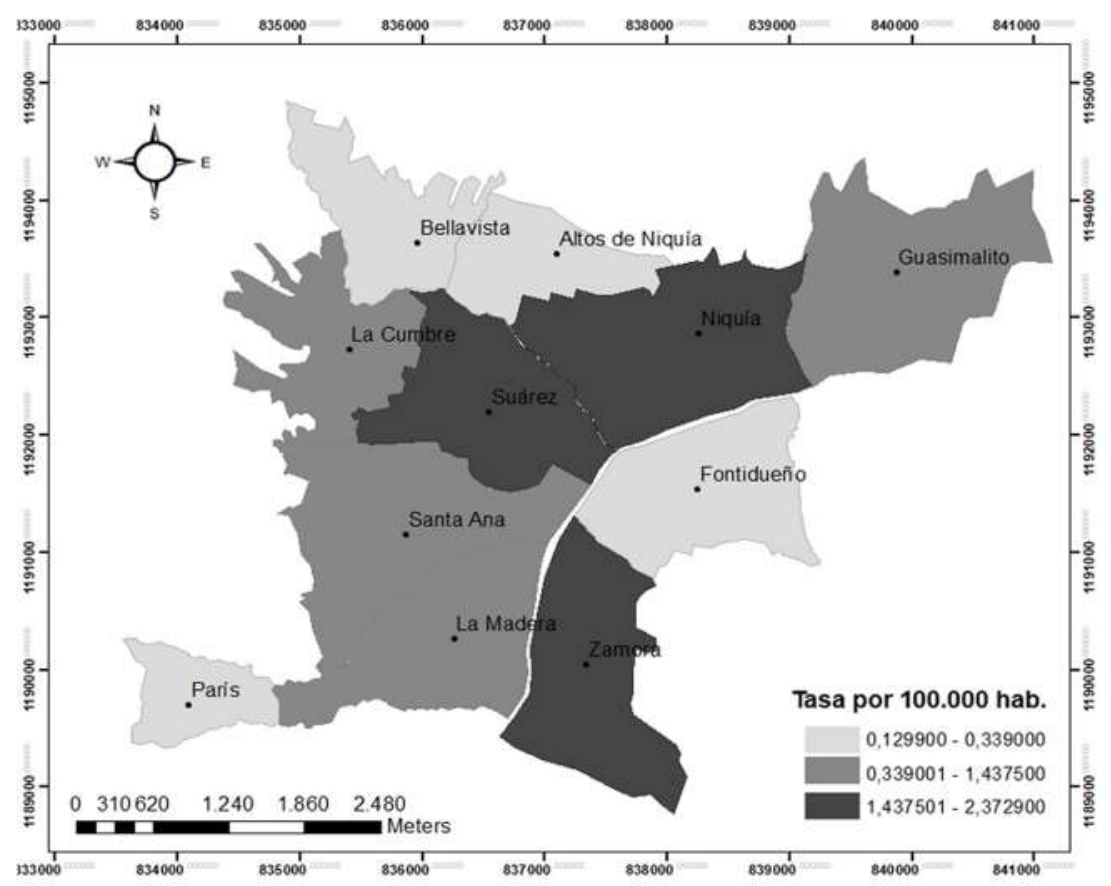

Figura 4. Tasa ajustada de mortalidad general por incidentes viales en las comunas del municipio de Bello, 2012-2016.

\section{Discusión}

En este artículo se presentan los resultados del estudio de la estructura general y geográfica de la mortalidad por incidentes viales, en el municipio de Bello, entre los años 2012 y 2016, con el fin de generar información que sirva para quienes toman las decisiones en salud pública y sustenten sus intervenciones.

En el quinquenio, se registraron 313 defunciones, en el municipio, por incidentes viales, lo que representó una tasa promedio de mortalidad del 14,0 por cada 100000 habitantes, un poco menos que la reportada en Colombia en el año 2016 por el Instituto Nacional de Medicina Legal y Ciencias Forenses, de 14,93 por cada 100000 habitantes, y que la de la región de las Américas, 15,9 por cada 100000 habitantes [7]. En este sentido, la oMs ha considerado los decesos en vialidades como una epidemia en la que Latinoamérica no es una excepción, con ciudades que exceden la tasa de la región, como son los casos de San Salvador (capital de El Salvador), con 75 decesos por cada 100000 habitantes; Campina Grande y Jôao Pessoa, ambas de Brasil, con tasas de 60,3 y 27,6, respectivamente; y Montería, Colombia, con 22,8 fallecimientos [21]. También los municipios de Villavicencio $(20,6)$, Pereira $(16,9)$ e Ibagué $(16,8)$ han tenido un importante protagonismo en el ámbito colombiano [21], lo que exige la revisión tanto de la normatividad como de las acciones e intervenciones, pues muy a pesar de la buena intención de las instancias que toman las decisiones en salud pública, los resultados son cuestionables.

En general, el estudio arrojó que fueron los hombres jóvenes los que tuvieron especial protagonismo en las defunciones. En un estudio sobre las pautas mundiales y regionales de la mortalidad entre la juventud, se evidenció que, entre los 10 y 24 años de edad, las lesiones generadas en los incidentes viales son una de las principales causas de mortalidad [22]. Al respecto, el Instituto de Tráfico y Seguridad Vial de España estableció que la incidentalidad en los adolescentes y jóvenes se debe a su actitud hacia el tráfico y la seguridad, a la poca formación vial, a la dependencia de las decisiones por parte un grupo y a que minimizan en menor medida el riesgo [23].

El hecho de que el mayor número de occisos por incidentes viales en Bello fueran jóvenes podría explicarse por la percepción que se tiene que estos son menos sensibles a los riesgos, sin pensar en las consecuencias, y parecen indiferentes a su propia seguridad La mayor presentación de las defunciones por incidentes viales en los fines de semana entre las 0:00 y 2:00 horas podría explicarse porque en ellos probablemente se incrementan los excesos nocturnos, en especial por parte de los jóvenes —asiduos noctámbulos-, lo que aunado con la disminución del flujo vehicular invita a que los conductores circulen a 
una velocidad mayor de la permitida y se convierta esto en una verdadera bomba de tiempo.

Es conocido que los conductores de motocicletas tienen un alto riesgo de sufrir lesiones fatales, ya que su cuerpo absorbe la energía que se disipa en el momento del incidente [24]. Es de resaltar que, en este estudio, el tipo de vehículo que más incidentalidad generó con desenlace fatal fue la motocicleta, lo que era de esperarse, dado que los conductores de este medio de transporte son los usuarios de la vía más vulnerables a la mortalidad, ya sea por choques con otros vehículos o con algún objeto fijo, afectando principalmente a los hombres entre 19 y 28 años de edad. Una razonable explicación de lo anterior estriba en el incremento desmesurado de este medio de transporte tanto en el municipio como en el país: en el año 2016, en el Registro Único Nacional de Tránsito se inventariarion cerca de 7 millones de motocicletas, cubriendo más del $55 \%$ de los vehículos registrados en el territorio nacional [7], lo que está relacionado con el hecho de que los hombres, en busca de un mejor estatus, y debido a requerimientos por asuntos laborales, usan con mayor frecuencia las motocicletas como medio de transporte, para realizar actividades cotidianas que facilitan la movilidad y mejoran la calidad de vida. No obstante, la conducción descuidada y la vulnerabilidad del conductor han llevado a un aumento en el número de incidentes que involucran motociclistas. En Brasil, los conductores de motocicletas son un grupo prioritario en los programas de prevención, porque en ellos es 7 veces más el riesgo de morir, 4 veces más probabilidad de sufrir lesiones y 2 veces más probabilidad de golpear a un peatón en comparación con los conductores de automóviles [25].

Los peatones ocuparon el segundo puesto con respecto a los usuarios más vulnerables de la vía, en el $42 \%$ de los casos, generados por un atropello de un automóvil, taxi, campero, camioneta, bus, buseta, microbús, camión, volqueta o tractocamión, y que afectó más a los adultos mayores. Este hallazgo podría explicarse debido a que esta población presenta procesos fisiológicos propios del envejecimiento, como la disminución en la agudeza visual, auditiva, retraso en el tiempo de reacción y la alteración del equilibrio y la marcha. Los hallazgos de esta investigación son afines a aquellos que se han descrito sobre la mortalidad por accidentes de tránsito en el adulto mayor en Colombia, donde las personas de 60 y más años tuvieron el doble de riesgo de morir por incidentes viales, por su alta vulnerabilidad y exposición a los factores de riesgo [26].

En general, prevalecieron las defunciones de personas entre 19 y 28 años de edad, especialmente conductores y pasajeros, por medio de un choque, si el tipo de vehículo impactado era una bicicleta o una motocicleta. Este hallazgo es similar al encontrado en Colombia en el año 2016, donde para los sujetos de mayor nivel educativo y edades entre 16 y 52 años se presentó más el choque con otros vehículos, dejando como víctimas fatales el conductor y el pasajero, con mayor participación de bicicletas, motocicletas o motocarros [27].

Los incidentes viales fueron más frecuentes en la Autopista Norte, específicamente en la diagonal 52, lo cual era de esperar, ya que por esta vía principal de entrada y salida del municipio se encuentra el peaje de Niquía (sur-norte), por el que diariamente transitan más de mil vehículos, y los controles en seguridad vial no logran cubrir esta cantidad en su totalidad, por lo que en algunos casos se presentan incidentes viales con víctimas fatales. A esto se suma que en esta zona, la vía carece de barandas para que las personas circulen, en las orillas de la carretera se ubican estaderos y discotecas que funcionan hasta altas horas de la noche, $y$ es probable que el consumo de sustancias que producen dependencia sea mayor, con el agravante que posibles estados de alicoramiento impidan que las personas no tomen las precauciones necesarias.

También cabe destacar que en la Autopista MedellínBogotá, ubicada en la comuna 11 (Zamora) del municipio de Bello, se presentó el $5,1 \%$ de las muertes por incidentes viales. Por esta vía, los vehículos transitan a altas velocidades en las horas de la noche, posiblemente por la confianza que les genera la disminución del trafico, lo que podría ser desencadenante de incidentes viales. Según el Observatorio Nacional de Seguridad Vial (20122017), "este es uno de los tramos a intervenir debido a la alta incidentalidad del sector" [28]. Tanto la Autopista Norte como la Autopista Medellín-Bogotá comunican al municipio con otros territorios que son altamente productivos en industria, comercio, turismo y agricultura.

Las comunas con más traumatismos fatales por incidentes viales en Bello fueron Suárez y Niquía. En Suárez se dio la tasa ajustada de mortalidad general más alta, probablemente porque en este lugar se encuentra la zona principal del municipio (Parque de Bello), donde confluyen varios centros comerciales, se presenta un alto flujo vehicular, y el comercio y el turismo son mayores. En Niquía, esta causa de muerte podría explicarse porque para los vehículos, en su paso por la glorieta de Niquía, ubicada en la diagonal 50 y 51 , no hay una señalización vial adecuada, lo que pone en riesgo el tránsito por esta vía; también porque allí se localiza uno de los centros comerciales más grande del municipio, donde el flujo de personas y vehículos es muy alto.

El panorama antes indicado no ha sido obstáculo para la continuidad de estrategias encaminadas a la disminución del flagelo de la incidentalidad vial por parte de la Secretaría de Movilidad del municipio: el ciudadano común ha observado avisos en vallas publicitarias, donde solicitan a la ciudadanía compromiso 
con la seguridad vial; se ha formalizado esta en públicos específicos, como peatones y conductores, con actividades de sensibilización para el cuidado de la vida, charlas sobre educación vial en puntos estrategicos de la ciudad, señalizacion en las calles para un mejor tránsito, medidas de barrera tipo maletín plástico, así como se han instalado grandes bolardos plásticos en las vías y se ha trabajado en el mejoramiento de algunas vías, entre otros. Se sugiere la persistencia en estas medidas y la valoración de su eficacia.

El barrio con la mortalidad más alta fue La Navarra, por donde queda el peaje de Niquía, zona ubicada en la Autopista Norte, específicamente en la diagonal 52, principal vía de incidentalidad en Bello.

Los medios de transporte terrestre son un apoyo al desarrollo social, económico y cultural de un país, pero también son causantes de una tragedia interminable, ya que son los sistemas más riesgosos y peligrosos a los que la humanidad se debe enfrentar diariamente [7]. El municipio de Bello contaba, para el año 2018, con el plan de movilidad para Niquía, que se esperaba permitiera la reorganización del flujo vehicular, estableciéndose un cierre temporal en sentido sur-norte de la rotonda, de la estación Niquia del Metro, de lunes a lunes, de 4:00 p. m. a 8:00 p. m. La calzada norte-sur se interviene con la puesta de barreras plásticas viales, con el objetivo de mejorar la circulación por esta vía, medidas que deberían beneficiar a la ciudadanía [29].

Además de las anteriores estrategias, las políticas, los planes, los programas, los proyectos, las actividades, entre otros, de la alcaldía municipal deberían concentrarse en mejorar la seguridad vial. Según el informe "La seguridad vial en la región de las Américas", de 2016, los aspectos a controlar serían: el consumo de alcohol y estupefacientes, la velocidad excesiva y la falta de uso del cinturón de seguridad y dispositivos de retención para los niños, mientras se maneja. La OMs establece que para una prevención eficaz y sostenible se exige de esfuerzos conjuntos que se concentren en contrarrestar tal flagelo [30].

La OMS y la ops coinciden en proponer la realización de intervenciones que minimicen la exposición a incidentes viales, como: ofrecer vehículos más resistentes al impacto, proporcionar caminos más cortos y seguros para los usuarios de la vía pública, especialmente los peatones, regular la conducción de los vehículos de motor a los jóvenes, planificar y diseñar vías más seguras, aplicar acciones correctoras en lugares de alta incidentalidad, mejorar el tiempo de respuesta de las ambulancias y atención en los hospitales, y sobre todo establecer normas más eficaces [4,5,31].

En conclusión, los hombres entre 19 y 28 años de edad presentaron las tasas más altas de mortalidad, especialmente si eran conductores o pasajeros de motocicletas, y cuya razón fue el choque con otro vehículo. Las mujeres mayores de 60 y más años fueron más vulnerables a este evento cuando eran peatonas, a causa de un atropello por un automóvil. La incidentalidad fue más prevalente en las vías que comunican al municipio con otros lugares (Autopista Norte y Autopista Medellín-Bogotá).

Debido al aumento de la incidentalidad vial en el municipio, se incrementaron las muertes por esta causa, por lo que es necesario realizar programas de prevención y promoción más contundentes y eficaces, para garantizarle a la ciudadanía un espacio vial más seguro, donde salir a cumplir con las actividades cotidianas sin verse involucrado en un incidente vial sea una realidad.

La principal limitación de esta investigación estuvo en las fuentes de información, ya que la administración del municipio no cuenta con unas bases de datos estandarizadas y el acceso a estas es muy restringido, por lo que dificulta realizar investigaciones sobre los diferentes indicadores epidemiológicos en salud.

Otra gran limitante fue la falta de estudios similares en el municipio de Bello, lo que impidió que se tuvieran referentes para la comparación de la magnitud de los indicadores.

De todos modos, se puede considerar este estudio como una línea de base y pionero en la municipalidad.

\section{Agradecimiento}

Agradecemos a:

La Alcaldía de Bello, por su apoyo en facilitar el trámite para la consecución de la información.

La Secretaría de Movilidad de Bello, por gestionarnos la adquisición de la información necesaria para la realización de la investigación.

El Grupo de Investigación Demografía y Salud, por las importantes sugerencias de sus integrantes para mejorar este producto.

Al ingeniero Alejandro Santamaría, por sus aportes para el análisis cartográfico y georreferenciado.

\section{Financiación}

La investigación se llevó a cabo con recursos propios.

\section{Conflicto de interés}

Los autores declaramos no tener conflicto de intereses.

\section{Declaración de responsabilidad}

Se declara que los puntos de vista expresados son responsabilidad de los autores y no de la institución en las que trabajan. 


\section{Referencias}

1. Colombia, Congreso de la República. Ley 769 del 2002, por la cual se expide el Código Nacional de Tránsito Terrestre y se dictan otras disposiciones (2002 agot. 6)

2. Bonilla J, Gutiérrez M. Injuries are not accidents: Towards a culture of prevention. Colombia Médica [internet]. 2014 [citado 2019 ago. 21]; 45(3). Disponible: http://colombiamedica.univalle. edu.co/index.php/comedica/article/view/1462/2427

3. Espinosa A, Cabrera G, Velásquez N. Epidemiología de incidentes viales en Medellín, Colombia, 2010-2015. Rev. Fac. Nac. Salud Pública [internet]. 2017 [citado 2019 agto. 21]; 35(1):7-15 DOI: https://doi.org/10.17533/udea.rfnsp.v35n1a02. Disponible en: http://www.scielo.org.co/pdf/rfnsp/v35n1/0120-386Xrfnsp-35-01-00007.pdf.

4. Organización Mundial de la Salud (OMS). Informe mundia sobre prevención de los traumatismos causados por el tránsito. Resumen. Ginebra: OMS [internet]; 2004. [citado 2017 sep. 13]. Disponible en: http://www.who.int/violence_injury_prevention/ publications/road_traffic/world_report/summary_es.pdf?ua $=1$.

5. Organización Mundial de la Salud (OMS). Las 10 principales causas de defunción [internet]. [citado 2018 jun. 13]. Disponible en: https:/www.who.int/es/news-room/fact-sheets/detail/the-top10-causes-of-death.

6. Organización Mundial de la Salud (OMS). Accidente de Tránsito [internet]. [citado 2019 ene. 13]. Disponible en: https://www.who. int/es/news-room/fact-sheets/detail/road-traffic-injuries.

7. Instituto Nacional de Medicina Legal y Ciencias Forenses. Forensis. Datos para la vida 2016. Bogotá: Instituto Nacional de Medicina Legal y Ciencias Forenses, Grupo Centro de Referencia Nacional sobre Violencia [internet]; 2017, 18(1). [citado 2017 sep. 14]. Disponible en: http://www.medicinalegal.gov.co/documents/20143/49526/Foren sis+2016.+Datos+para+la+vida.pdf.

8. Asamblea General de las Naciones Unidas. Plan mundial para el decenio de acción para la seguridad vial 2011-2020 [internet]; 2011 [citado 2017 sep. 16]. Disponible en: http://www.who.int/ roadsafety/decade_of_action/plan/spanish.pdf.

9. Colombia, Ministerio de Transporte. Plan Nacional de Seguridad Vial Colombia 2011-2021. 2. ${ }^{\mathrm{a}}$ ed. [internet]; 2015 [citado 2017 sep. 16]. Disponible en: https://ansv.gov.co/public/documentos/ PLAN\%20NACIONAL\%20DE\%20SEGURIDAD\%20VIAL. compressed.pdf.

10. Organización Mundial de la Salud (OMS). Informe sobre la situación mundial de la seguridad vial 2015 [internet]; 2015 [citado 2017 sep. 14]. Disponible en: http://www.who.int/ violence_injury_prevention/road_safety_status/2015/Summary_ GSRRS2015_SPA.pdf?ua=1.

11. Alcaldía de Bello. Plan de Desarrollo 2016-2019. "Bello, ciudad de progreso" [internet]. s. f. [citado 2018 feb. 15]. Disponible en: https://bello.gov.co/index.php/features/nuestros-planes/item/dow nload/81_6601676e 0dbcd77c1f5d0ff1dd787bcb

12. Organización Panamericana de la Salud (OPS). Clasificación estadística internacional de enfermedades y problemas relacionados con la salud. Décima Revisión. CIE-10 [internet] 2003 [citado 2018 mar. 11]. Disponible en: http://iris.paho.org/ xmlui/bitstream/handle/123456789/6282/Volume1.pdf

13. Organización Panamericana de la Salud (OPS), Organización Mundial de la Salud (OMS). Lista 6/67 - CIE-10 OMS/OPS [internet]; s. f. [citado 2018 mar. 15]. Disponible en: https://www. minsalud.gov.co/sites/rid/Lists/BibliotecaDigital/RIDE/INEC/ INTOR/8.Lista_Mort\%20OPS\%20667.pdf.
14. ArcGis Pro. Densidad Kernel [internet]. s. f. [citado 2018 mar. 11]. Disponible en: https://pro.arcgis.com/es/pro-app/tool-reference/ spatial-analyst/kernel-density.htm.

15. ArcGis. Clasificar campos númericos para simbología graduada [internet]; 2012 [citado 2018 mar. 15]. Disponible en: $\quad$ http://help.arcgis.com/es/arcgisdesktop/10.0/help/index. html\#//00s50000001r000000.

16. Colombia, Ministerio de Salud. Resolución 8430, por la cual se establecen las normas científicas, técnicas y administrativas para la investigación en salud (1993 oct. 4).

17. Colombia, Congreso de la República. Ley Estatutaria 1581, por la cual se dictan disposiciones generales para la protección de datos personales (2012 oct. 18).

18. Colombia, Ministerio de Comercio, Industria y Turismo. Decreto 1317, por el cual se reglamenta parcialmente la Ley 1581 de 2012 (2013 jun. 27).

19. Ministerio de Salud. Ciclo de vida [internet]. s. f. [citado 2018 jun. 22]. Disponible en: https://www.minsalud.gov.co/ proteccionsocial/Paginas/cicloVida.aspx

20. Real Academia Española. "Atropellar" y "Chocar". Recuperado de https://dle.rae.es/?id=4Lwwaty y https://dle.rae. es $/$ ? id $=8$ w40REN.

21. LA Network. Ranking latinoamericano de ciudades fatales. Mortalidad vial urbana [internet]. s. f. [citado 2019 jun. 22]. Disponible en: https://a.network/wp-content/uploads/2017/12/ CIUDADES-FATALES-FINAL.pdf.

22. Organización Mundial de la Salud (OMS). Los accidentes de tránsito, el suicidio y las afecciones maternas figuran entre las principales causas de muerte de los jóvenes [internet]; 2009 sep. 11 [citado 2018 abr. 25]. Disponible en: http:// www.who.int/mediacentre/news/releases/2009/adolescent mortality_20090911/es/.

23. Universidad de Valencia, Instituto de Tráfico y Seguridad Vial. La edad, factor clave en los accidentes de tráfico (2000-2004) [internet]. s. f. [citado 2018 abr. 25]. Disponible en: https:// www.lineadirecta.com/ZZRecursos/recursos/ES/documentos/ GruposdeRiesgo06.pdf.

24. Aristizábal D, González G, Suárez J, et al. Factores asociados al trauma fatal en motociclistas en Medellín, 2005-2008. Biomédica. 2012;32(1):112-24. DOI: https://doi.org/10.7705/biomedica. v32i1.603.

25. Bacchieri G, Barros A. Traffic accidents in Brazil from 1998 to 2010: Many changes and few effects. Rev. Saúde Pública [internet]. 2011 [citado 2018 abr. 25]; 45(5):1-14. DOI: http:// dx.doi.org/10.1590/S0034-89102011005000069. Disponible en: http://www.scielo.br/scielo.php?script=sci_arttext\&pid=S0034$89102011005000069 \& \operatorname{lng}=\mathrm{en} \& \mathrm{nrm}=\mathrm{iso} \& \operatorname{tln} \mathrm{g}=\mathrm{en}$.

26. Segura Cardona AM, Cardona Arango D, Berbesí Fernández DY, et al. Mortalidad por accidente de tránsito en el adulto mayor en Colombia. Rev. Saúde Pública [internet]. 2017[citado 2018 abr. 25]; 51:1-8. DOI: http://dx.doi.org/10.1590/s15188787.2017051006405. Disponible en: http://www.scielo.br/pdf/ rsp/v51/es_0034-8910-rsp-S1518-87872017051006405.pdf.

27. Ruiz J, Herrera A. Accidentes de tránsito con heridos en Colombia según fuentes de información: caracterización general y tipologías de accidentes. CES Psicol [internet]. 2016 [citado 2018 abr. 25]; 9(1):32-46. Disponible en: http://www.scielo.org.co/pdf/cesp/ v9n1/v9n1a04.pdf.

28. Observatorio Nacional de Seguridad Vial. Plan de control operativo 2018 [internet]. [citado 2018 abr. 25]. Disponible en: https://ansv.gov.co/observatorio/?op=Contenidos\&sec $=74$ 
29. Alcaldia de Bello. Bello presenta plan de movilidad para Niquía [internet]. 2017 [citado 2018 abr. 26]. Disponible en: http://www. bello.gov.co/index.php/component/k2/item/370movilidad-paraniquia.

30. Organización Panamericana de la Salud, Organización Mundial de la Salud. La seguridad vial en la región de las Américas [internet]; 2016 [citado 2017 oct. 25]. Disponible en: http://iris.paho.org/ xmlui/bitstream/handle/123456789/28565/9789275319123-spa pdf? sequence $=6$.

31. Organización Panamericana de la Salud. Factores de riesgo en la seguridad vial [internet]. 2004 abr. 7 [citado 2017 oct. 16]. Disponible en: http://www.paho.org/bol/index.php?option=com content\&view=article\&id=299: factores-riesgo-seguridadvial\&Itemid=481. 